\title{
Strongly Hopfian and Strongly Cohopfian Objects in the Category of Complexes of Left A-Modules
}

\author{
El Hadj Ousseynou Diallo ${ }^{1}$, Mohamed Ben Faraj Ben Maaouia ${ }^{2}$ \& Mamadou Sangharé ${ }^{1}$ \\ ${ }^{1}$ Déartement de Mathéatique, Universite Cheikh Anta Diop, Dakar, Senegal \\ ${ }^{2}$ Unité de Formation et de Recherche de Sciences Appliquées et de Technologie, Universite Gaston Berger, Saint- \\ Louis, Senegal \\ Correspondence: Mohamed Ben Faraj Ben Maaouia, Unité de Formation et de Recherche de Sciences Appliquées \\ et de Technologie, Universite Gaston Berger, Saint-Louis, Senegal. Tel: 221-77-519-0203. E-mail: \\ maaouiaalg@hotmail.com
}

Received: December 5, 2013 Accepted: April 1, 2014 Online Published: July 7, 2014

doi:10.5539/jmr.v6n3p81 URL: http://dx.doi.org/10.5539/jmr.v6n3p81

\section{Abstract}

The object of this paper is the study of strongly hopfian, strongly cohopfian, quasi-injective, quasi-projective, Fitting objects of the category of complexes of $A$-modules.

In this paper we demonstrate the following results:

a)If $C$ is a strongly hopfian chain complex (respectively strongly cohopfian chain complex) and $E$ a subcomplex which is direct summand then $E$ and $C / E$ are both strongly Hopfian (respectively strongly coHopfian) then $C$ is strongly Hopfian (respectively strongly coHopfian).

b)Given a chain complex $C$, if $C$ is quasi-injective and strongly-hopfian then $C$ is strongly cohopfian.

c)Given a chain complex $C$, if $C$ is quasi-projective and strongly-cohopfian then $C$ is strongly hopfian.

In conclusion the main result of this article is the following theorem:

Any quasi-projective and strongly-hopfian or quasi-injective and strongly-cohofian chain complex of $A$-modules is a Fitting chain complex.

Keywords: chain complex, strongly-hopfian, strongly-cohopfian, quasi-injective, quasi-projective, fitting chain complex

\section{Introduction}

In this paper, we will study strongly hopfian, strongly cohopfian, quasi-injective, quasi-projective objects of the category of complexes of $A$-modules denoted by $C O M P$.

We also study Fitting objects of the category of complexes of $A$-modules.

The objects of COMP are chain complexes and the morphisms are maps of chains.

A chain complex $(C, d): \ldots \rightarrow C_{n+1} \stackrel{d_{n+1}}{\rightarrow} C_{n} \stackrel{d_{n}}{\rightarrow} C_{n-1} \stackrel{d_{n-1}}{\rightarrow} \ldots$ is a sequence $\left(d_{n}\right)$ of homomorphisms of left $A$-modules verifying $d_{n} \circ d_{n+1}=0$, for all $n \in \mathbf{Z}$.

A chain map $f$ of $(C, d)$ into $\left(C^{\prime}, d^{\prime}\right)$ is defined by:

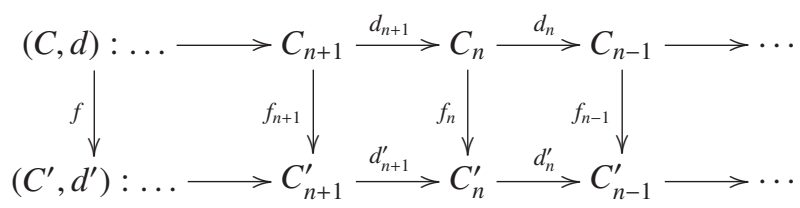

with $d_{n+1}^{\prime} \circ f_{n+1}=f_{n} \circ d_{n+1}, \forall n \in \mathbb{Z}$.

Given $(C, d)$ an object of $C O M P$ and $f$ an endomorphism of $(C, d)$. 
$C$ is said to be hopfian (respectively cohopfian) if any epimorphism (respectively monomorphism) is an isomorphism.

A chain complex $(C, d): \ldots \rightarrow C_{n+1} \stackrel{d_{n+1}}{\rightarrow} C_{n} \stackrel{d_{n}}{\rightarrow} C_{n-1} \stackrel{d_{n-1}}{\rightarrow} \ldots$ is said to be fully invariant if and only if each $A-$ module $C_{n}$ is fully invariant, $\forall n \in \mathbb{Z}$.

A chain complex $(C, d): \ldots \rightarrow C_{n+1} \stackrel{d_{n+1}}{\rightarrow} C_{n} \stackrel{d_{n}}{\rightarrow} C_{n-1} \stackrel{d_{n-1}}{\rightarrow} \ldots$ is said essential if and only if each $A-$ module $C_{n}$ is essential, $\forall n \in \mathbb{Z}$.

A chain complex $(C, d)$ is said Fitting if for any endomorphism $f$ of $(C, d)$, there exists an integer $n$ such $C=$ $\operatorname{Im} f^{n} \oplus \operatorname{Ker} f^{n}$.

After $(C, d)$ is noted by $C$.

In this paper we demonstrate the following results:

a) If $C$ is a strongly hopfian chain complex (respectively strongly cohopfian chain complex) and $E$ a subcomplex is direct summand then $E$ and $C / E$ are both strongly Hopfian (respectively strongly coHopfian) then $C$ is strongly Hopfian (respectively strongly coHopfian).

b) If $E$ is a fully invariant chain complex such that $E$ and $C / E$ are strongly Hopfian (respectively strongly coHopfian) then $C$ is strongly Hopfian (respectively strongly coHopfian).

c) Given $C=\oplus C^{i}$ such $C^{i}$ is fully invariant, if $C^{i}$ is a strongly hopfian (respectively strongly cohopfian) chain complex then $C$ is a strongly hopfian (respectively strongly cohopfian) chain complex.

d) Given a chain complex $C: \ldots \rightarrow C_{n+1} \stackrel{d_{n+1}}{\rightarrow} C_{n} \stackrel{d_{n}}{\rightarrow} C_{n-1} \stackrel{d_{n-1}}{\rightarrow} \ldots, C$ is quasi-injective, if and only if, $C_{n}$ is quasi - injective, for all $n \in \mathbb{Z}$.

e) A chain complex $C: \ldots \rightarrow C_{n+1} \stackrel{d_{n+1}}{\rightarrow} C_{n} \stackrel{d_{n}}{\rightarrow} C_{n-1} \stackrel{d_{n-1}}{\rightarrow} \ldots$, is quasi-projective, if and only if, $C_{n}$ is quasiprojective, for all $n \in \mathbb{Z}$.

f) Given a chain complex $C$, if $C$ is quasi-injective and strongly- hopfian then $C$ is strongly cohopfian.

g) Given a chain complex $C$, if $C$ is quasi-projective and strongly- cohopfian then $C$ is strongly hopfian.

h) Any quasi-projective and strongly-hopfian or quasi-injective and strongly-cohofian chain complex of A-modules is a Fitting chain complex.

In this paper $A$ denotes a not inevitably commutative, unitarian associative $\operatorname{ring}$ and $M$ a left unifere module.

\section{Definitions and Preliminary Results on the Category COMP}

Definition 1 Given $(C, d)$ a chain complex of left $A$ modules and $f$ an endomorphism of $(C, d)$ such that:

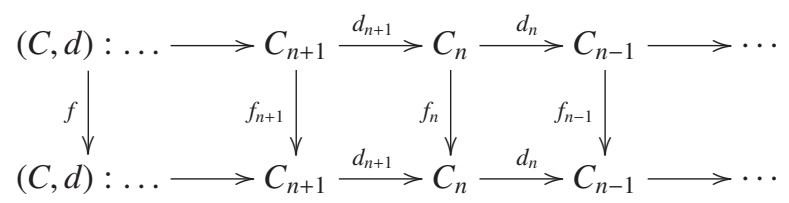

We call $f \circ f$ the chain map compounded of $f$ by itself denoted $f^{2}$.

We also define $\left(f^{2}\right)_{n}=f_{n} \circ f_{n}$, for all $n \in \mathbb{Z}$ :

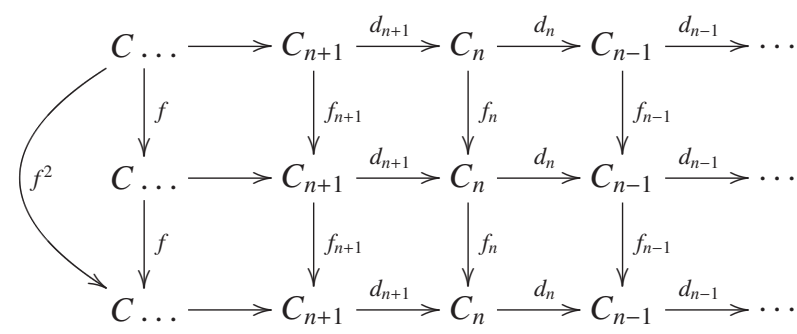

We also define $f^{k}$ such $f_{n}^{k}=f_{n} \circ f_{n} \circ \ldots \circ f_{n}$, with $k$ factors, for all $n \in \mathbb{Z}$. 
Proposition 1 Considering $(C, d)$ a chain complex of $A$ - modules and $f$ an endomorphism of $(C, d)$ :

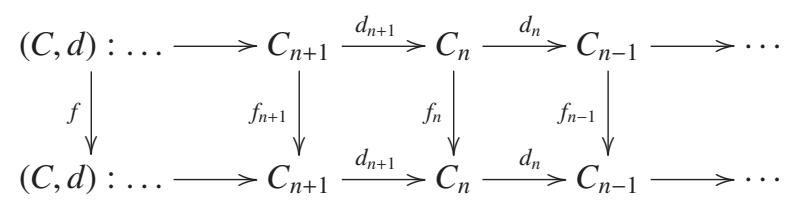

Given

$$
\begin{aligned}
\Delta_{n+1}^{k}: \quad \operatorname{Kerf}_{n+1}^{k} & \rightarrow \operatorname{Kerf}_{n}^{k} \\
x & \mapsto d_{n+1}(x)
\end{aligned}
$$

where $f_{n+1}^{k}=f_{n+1} \circ f_{n+1} \circ \ldots \circ f_{n+1}$ with $k$ factors, then $\Delta_{n+1}^{k}$ is the induced morphism by $\Delta_{n+1}^{k+1}$.

Proof. Considering

$$
\begin{aligned}
\Delta_{n+1}^{k}: \operatorname{Kerf}_{n+1}^{k} & \rightarrow \operatorname{Kerf}_{n}^{k} \\
x & \mapsto d_{n+1}(x)
\end{aligned}
$$

and

$$
\begin{aligned}
\Delta_{n+1}^{k+1}: \quad \operatorname{Kerf}_{n+1}^{k+1} & \rightarrow \operatorname{Kerf}_{n}^{k+1} \\
x & \mapsto d_{n+1}(x) .
\end{aligned}
$$

We obtain $\operatorname{Kerf}_{n+1}^{k} \subseteq \operatorname{Ker} f_{n+1}^{k+1}$ et $\operatorname{Kerf} f_{n}^{k} \subseteq \operatorname{Ker} f_{n}^{k+1}$ therefore $\Delta_{n+1}^{k}$ is the induced by $\Delta_{n+1}^{k+1}$.

Proposition 2 Let be $(C, d)$ a chain complex of A-modules and $f$ an endomorphism of $(C, d)$ such that:

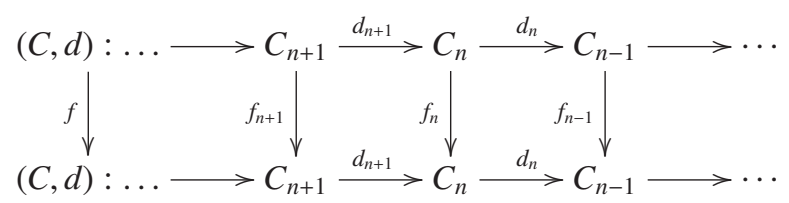

Given

$$
\begin{aligned}
\delta_{n+1}^{k}: \quad \operatorname{Im} f_{n+1}^{k} & \rightarrow \quad \operatorname{Im} f_{n}^{k} \\
x & \mapsto d_{n+1}(x)
\end{aligned}
$$

Then $\delta_{n+1}^{k+1}$ is the induced morphism by $\delta_{n+1}^{k}$.

Proof. Given

$$
\begin{aligned}
\delta_{n+1}^{k}: \quad \operatorname{Im} f_{n+1}^{k} & \rightarrow \quad \operatorname{Im} f_{n}^{k} \\
x & \mapsto d_{n+1}(x)
\end{aligned}
$$

and

$$
\begin{aligned}
\delta_{n+1}^{k}: \operatorname{Im}_{n+1}^{k+1} & \rightarrow \operatorname{Im} f_{n}^{k+1} \\
x & \mapsto d_{n+1}(x)
\end{aligned}
$$

then $\operatorname{Im} f_{n+1}^{k+1} \subseteq \operatorname{Im} f_{n+1}^{k}$ et $\operatorname{Im} f_{n}^{k+1} \subseteq \operatorname{Im} f_{n}^{k}$ d'o $\delta_{n+1}^{k+1}$ is the morphism induced by $\delta_{n+1}^{k}$.

Definition 2 Let $C: \ldots \rightarrow C_{n+1} \stackrel{d_{n+1}}{\rightarrow} C_{n} \stackrel{d_{n}}{\rightarrow} C_{n-1} \stackrel{d_{n-1}}{\rightarrow} \ldots$ a chain complex and $E: \ldots \rightarrow E_{n+1} \stackrel{u_{n+1}}{\rightarrow} E_{n} \stackrel{u_{n}}{\rightarrow} E_{n-1} \stackrel{u_{n-1}}{\rightarrow} \ldots$ a subcomplex of $C$. Then $E$ is direct summand, if and only if, $E_{n}$ is direct summand.

Definition 3 Let $N$ a sub-module of a $A$ - module $M$. Then $N$ is said to be fully invariant, if for any endomorphism $f$ of $M$ we have $f(N) \subseteq N$.

Definition 4 Given $C: \ldots C_{n+1} \rightarrow C_{n} \rightarrow C_{n-1} \rightarrow \ldots$ a chain complexes of $A$ - modules and $E: \ldots E_{n+1} \rightarrow E_{n} \rightarrow$ $E_{n-1} \rightarrow \ldots$ a subcomplex of $C$. Then $E$ is said to be subcomplex fully invariant in $C$, if for all $n \in \mathbb{Z}, E_{n}$ is fully invariant. 
3. Strongly Hopfian Objects and Strongly CoHopfian Objects of the Category of Complexes of Left $A$ Modules

\subsection{Strongly Hopfian and Strongly CoHopfian}

Definition 5 Let be $f$ an endomorphism of chain complex $(C, d)$ :

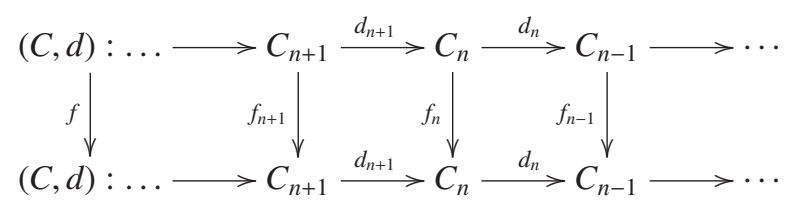

Then:

a) the chain complex

$$
\left(\operatorname{Kerf}^{k}\right): \ldots \rightarrow \operatorname{Kerf}_{n+1}^{k} \stackrel{\Delta_{n+1}^{k}}{\rightarrow} \operatorname{Kerf}_{n}^{k} \stackrel{\Delta_{n}^{k}}{\rightarrow} \operatorname{Kerf}_{n-1}^{k} \stackrel{\Delta_{n-1}^{k}}{\rightarrow} \ldots
$$

stabilizes, if and only if, it exists $k_{0} \in \mathbb{N}^{\star} \operatorname{such}$ that $\left(\operatorname{Ker} f^{k_{0}}\right)=\left(\operatorname{Kerf} f^{k_{0}+s}\right)$, for all $s \in \mathbb{N}$.

b) the chain complex

$$
\left(\operatorname{Im} f^{k}\right): \ldots \rightarrow \operatorname{Im} f_{n+1}^{k} \stackrel{\delta_{n+1}^{k}}{\rightarrow} \operatorname{Im} f_{n}^{k} \stackrel{\delta_{n}^{k}}{\rightarrow} \operatorname{Im} f_{n-1}^{k} \stackrel{\delta_{n-1}^{k}}{\rightarrow} \ldots
$$

stabilizes if it exists $k_{0} \in \mathbb{N}^{\star}$ such that $\left(\operatorname{Im} f^{k_{0}}\right)=\operatorname{Im} f^{k_{0}+s}$, for all $s \in \mathbb{N}$.

Proposition 3 Given $f$ an endomorphism of a chain complex $(C, d)$ :

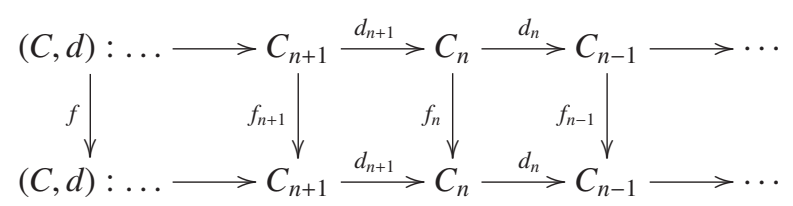

Then $\left(\operatorname{Kerf}^{k}\right)$ stabilizes if and only if $\left(\operatorname{Ker} f_{n}^{k}\right)$ stabilizes for all $n \in \mathbb{Z}$.

Proof. If $\left(K e r f^{k}\right)$ stabilizes then it exists $k_{0} \in \mathbb{N}^{\star}$ such that $\left(\operatorname{Kerf} f^{k_{0}}\right)=\left(\operatorname{Ker} f^{k_{0}+s}\right)$, for all $s \in \mathbb{N}$ hence $\operatorname{Ker} f_{n}^{k_{0}}=$ $\operatorname{Kerf} f_{n}^{k_{0}+s}$ for all $n \in \mathbb{Z}$. This implies that que $\left(\operatorname{Ker} f_{n}^{k}\right)$ stabilizes for all $n \in \mathbb{Z}$.

Reciprocally suppose that $\left(\operatorname{Ker} f_{n}^{k}\right)$ stabilizes for all $n \in \mathbb{Z}$ then it exists $k_{0} \in \mathbb{N}^{\star}$ such that for all $s \in \mathbb{N}$ then $\operatorname{Ker} f_{n}^{k_{0}}=\operatorname{Kerf}_{n}^{k_{0}+s}$ for all $n \in \mathbb{Z}$.

So $\left(K e r f^{k}\right)$ stabilizes for all positive integer $k$.

Proposition 4 Given $f$ an endomorphism of a chain complex $(C, d)$ :

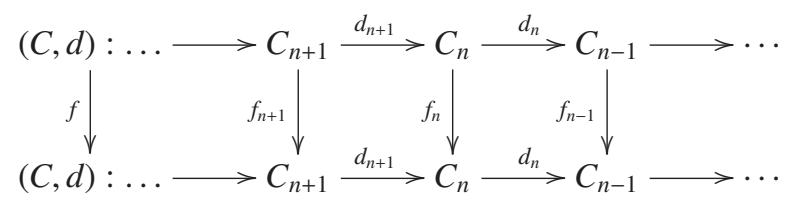

Then $\left(\operatorname{Im} f^{k}\right)$ stabilizes if and only if $\left(\operatorname{Im} f_{n}^{k}\right)$ stabilizes for all $n \in \mathbb{Z}$.

Proof. Suppose $\left(\operatorname{Im} f^{k}\right)$ stabilizes then it exists $k_{0} \in \mathbb{N}^{\star}$ such that $\left(\operatorname{Im} f^{k_{0}}\right)=\left(\operatorname{Im} f^{k_{0}+s}\right)$, for all $s \in \mathbb{N}$ hence $\operatorname{Im} f_{n}^{k_{0}}=\operatorname{Im} f_{n}^{k_{0}+s}$ for all $n \in \mathbb{Z}$. Hence $\left(\operatorname{Im} f_{n}^{k}\right)$ stabilizes for all $n \in \mathbb{Z}$.

Reciprocally suppose that $\left(\operatorname{Im} f_{n}^{k}\right)$ stabilizes for all $n \in \mathbb{Z}$ then there is $k_{0} \in \mathbb{N}^{\star}$ such that for all $s \in \mathbb{N}$ so $\operatorname{Im} f_{n}^{k_{0}}=$ $\operatorname{Im} f_{n}^{k_{0}+s}$ for all $n \in \mathbb{Z}$. That proove $\left(\operatorname{Im} f^{k}\right)$ stabilizes for all $k$.

Definition 6 An $A$ - module $M$ is said to be strongly hopfian (respectively strongly cohopfian) if for any endomorphism $f$ of $M$ the sequence $\left(\operatorname{Ker} f^{n}\right)$ stabilizes: $\operatorname{ker} f^{2} \subseteq \operatorname{ker} f^{3} \ldots \subseteq \operatorname{Ker} f^{n}$ (respectively $\operatorname{Imf} \supseteq \operatorname{Im} f^{2} \ldots \supseteq$ $\left.\operatorname{Im} f^{n} \ldots\right)$.

Definition 7 A chain complex $C$ of $A$-modules is said to be strongly hopfian(respectively strongly cohopfian), if for any endomorphism $f$ of $(C, d),\left(\operatorname{Ker} f^{k}\right)$ (respectively $\left.\left(\operatorname{Im} f^{k}\right)\right)$ stabilizes. 
Proposition 5 A chain complex $C$ is strongly hopfian if it exists $k \in \mathbb{N}$ such that $\operatorname{Ker} f_{n}^{k} \cap \operatorname{Im} f_{n}^{k}=0$, for all $n \in \mathbb{Z}$.

Proof. Using the Proposition 2.5 (see Hmaimou, 2007) and let be $k \in \mathbb{N}$ such that $\operatorname{Ker} f_{n}^{k} \cap \operatorname{Im} f_{n}^{k}=0$ then $\left(\operatorname{Ker} f_{n}^{k}\right)$ stabilizes for all $n \in \mathbb{Z}$. That prove $\left(K e r f^{k}\right)$ stabilizes, so $C$ is a strongly hopfian chain complex.

Proposition 6 A chain complex $C$ :

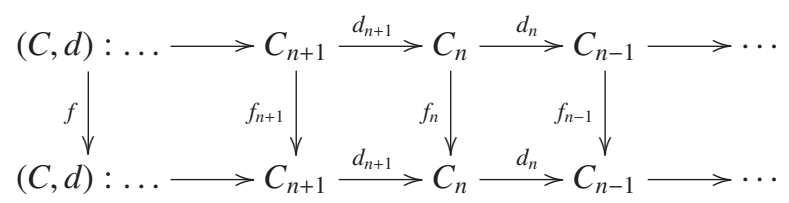

is strongly cohopfian, if it exists a positive integer $k$ such that:

$$
\operatorname{Im} f_{n}^{k}+\operatorname{Ker} f_{n}^{k}=C_{n}, \text { for all } n \in \mathbb{Z} .
$$

Proof. Using the Proposition 2.6 (see Hmaimou, 2007), we can suppose it exists a positive integer $k$ such that: $\operatorname{Im} f_{n}^{k}+\operatorname{Ker} f_{n}^{k}=C_{n}$, for all $n \in \mathbb{Z}$ then $\left(\operatorname{Im} f_{n}^{k}\right)$ stabilizes for all $n \in \mathbb{Z}$ hence $\left(\operatorname{Im} f^{k}\right)$ stabilizes so $C$ is a strongly cohopfian complex.

Theorem 1 Considering $C$ a chain complex and $E$ a subcomplex of $C$. If $C$ is strongly hopfian (respectively is strongly cohopfian) and $E$ is direct summand then $E$ et $C / E$ both are strongly hopfian (respectively strongly cohopfian).

Proof. Let us demonstrate at first that $E$ is strongly hopfian (respectively strongly cohopfian).

Suppose $C=E \oplus K$ and let be $g$ a chain map of $E$ in itself which can be extended to $C$ such as $f=g \oplus 0$, with 0 the zero morphism of $K$.

Given $C$ is strongly hopfian (respectively strongly cohopfian), then $\left(\operatorname{Ker} f^{k}\right)$ (respectively $\left.\left(\operatorname{Im} f^{k}\right)\right)$ stabilizes then it exists $k_{0} \in \mathbb{N}^{\star}$ such that $\left(\operatorname{Ker} f^{k_{0}}\right)=\left(\operatorname{Ker} f^{k_{0}+s}\right)\left(\operatorname{respectively}\left(\operatorname{Im} f^{k_{0}}\right)=\left(\operatorname{Im} f^{k_{0}+s}\right)\right)$, for all $s \in \mathbb{N}$, then $\operatorname{Ker} f_{n}^{k_{0}}=$ $\operatorname{Ker} f_{n}^{k_{0}+s}$ (respectively $\operatorname{Im} f_{n}^{k_{0}}=\operatorname{Im} f_{n}^{k_{0}+s}$ pour tout $n \in \mathbb{Z}$ ), therefore $C_{n}$ is strongly hopfian (respectively strongly cohopfian) and $E_{n}$ is direct summand.

Hence $C_{n}$ and $C_{n} / E_{n}$ for all $n \in \mathbb{Z}$ are both strongly hopfian (respectively strongly cohopfian).

That prove $E$ and $C / E$ are strongly hopfian (respectively strongly cohopfian).

Theorem 2 Considering $C$ a chain complex and $E$ a subcomplexe of $C$, if $E$ is fully invariant with $E$ and $C / E$ both strongly hopfian (respectively strongly cohopfian) then $C$ is strongly hopfian (respectively strongly cohopfian).

Proof. Let be $(C, d)$ a chain complex of $A$-modules and $f$ an endomorphism of $(C, d)$ verifying:

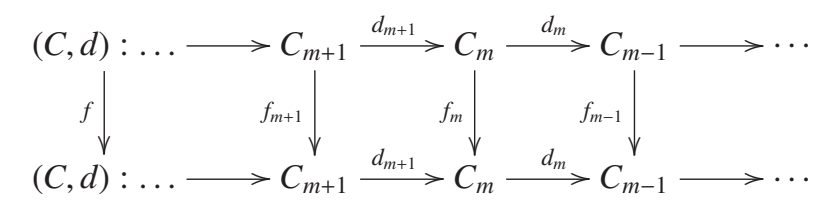

Given $E$ is fully invariant in $C$ then $f$ induces a chain map $h$ of $E$ in itself such that:

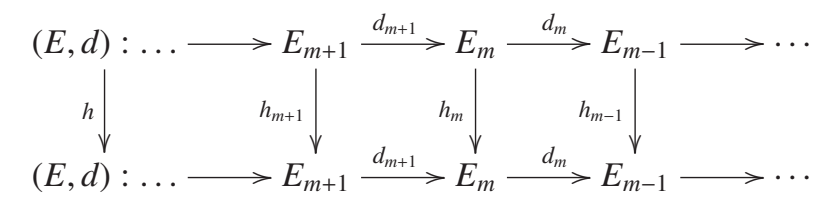

$f$ induces also a chain map g of $C / E$ in itself such that:

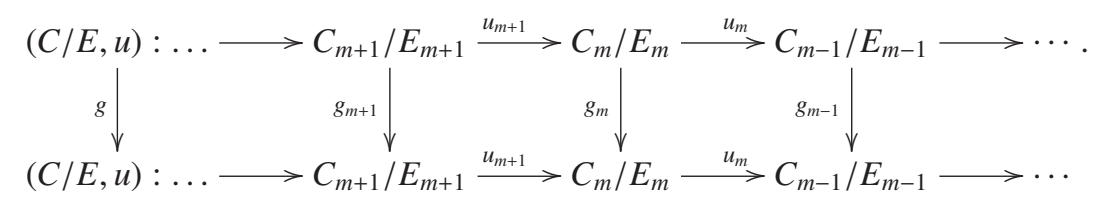


Given $E$ and $C / E$ are both strongly cohopfian, hence $I m h^{n}=I m h^{n+k}$ and $I m g^{n}=I m g^{n+k}$, therefore for all $m \in \mathbb{Z}$, we have $I m h_{m}^{n}=I m h_{m}^{n+k}$ and $I m g_{m}^{n}=I m g_{m}^{n+k}$.

If $p=2 n$, for $x \in C_{m}$, we obtain $g_{m}^{n}\left(x+E_{m}\right)=g_{m}^{n+1}\left(y+E_{m}\right)$ for some $y \in E_{m}$. Then $t=f_{m}^{n}(x)-f_{m}^{n}(y) \in E_{m}$, hence $f_{m}^{n}(t)=f_{m}^{p+1}(z)$ for some $z \in E_{m}$, therefore $f_{m}^{p}(x)=f_{m}^{p+1}(y+z)$, so $C$ is strongly cohopfian.

Suppose that $E$ and $C / E$ are both hopfian, then $\operatorname{Kerh}^{n}=\operatorname{Kerh}^{n+k}$ and $\operatorname{Kerg}^{n}=\operatorname{Kerg}^{n+k}$, hence for all $m \in \mathbb{Z}$, we have $\operatorname{Kerh}_{m}^{n}=\operatorname{Kerh}_{m}^{n+k}$ and $\operatorname{Kerg}_{m}^{n}=\operatorname{Kerg}_{m}^{n+k}$.

Let be $x \in \operatorname{Ker} f_{m}^{2 n}$, then $g^{2 n+1}\left(x+E_{m}\right)=0$, so $y=f_{m}^{n}(x) \in E_{m}$ and $f_{m}^{n+1}(y)=0$. Therefore $y \in \operatorname{Kerh}_{m}^{n+1}=\operatorname{Kerh}_{m}^{n}$, then $x \in \operatorname{Kerf}_{m}^{2 n}$, so $C$ is strongly hopfian.

Corollary 1 Let be $C=\oplus C^{i}$ where $C^{i}$ is a subcomplex fully invariant of $C$ for all $i \in I$. If $C^{i}$ is strongly hopfian (respectively strongly cohopfian), then $C$ is strongly hopfian (respectively strongly cohopfian).

Proof. Let be $f$ an endomorphism of chain complex $C$. Then it exists a unique family $\left(f^{i}\right)$ such that $i \in I$ where $f^{i}=\left.f\right|_{C^{i}}$ and $m=\sum n_{i} . f^{i}$ is denoted by $\left[f^{i}\right] . C^{i}$ is strongly hopfian then $\operatorname{Ker}\left[f^{i}\right]^{n_{i}}=\operatorname{Ker}\left[f^{i}\right]^{n_{i}+1}$, therefore $\oplus \operatorname{Ker}\left[f^{i}\right]^{m}=\oplus \operatorname{Ker}\left[f^{i}\right]^{n_{i}+1}$. So $\operatorname{Ker}\left(\oplus\left[f^{i}\right]\right)^{m}=\operatorname{Ker}\left(\oplus\left[f^{i}\right]\right)^{m+1}$, then $C$ is strongly hopfian.

Let be $f$ an endomorphism of $C$. Then it exists a unique family $\left(f^{i}\right)$ where $i \in I f^{i}=\left.f\right|_{C^{i}}$ and $m=\sum n_{i}$. $C^{i}$ is strongly cohopfian, then $\operatorname{Im}\left[f^{i}\right]^{n_{i}}=\operatorname{Im}\left[f^{i}\right]^{n_{i}+1}$, therefore $\oplus \operatorname{Im}\left[f^{i}\right]^{m}=\oplus \operatorname{Im}\left[f^{i}\right]^{n_{i}+1}$. So $\operatorname{Im}\left(\oplus\left[f^{i}\right]\right)^{m}=\operatorname{Im}\left(\oplus\left[f^{i}\right]\right)^{m+1}$, then $C$ is strongly cohopfian.

\subsection{Quasi-Injective Chain Complex}

Definition 8 An $A$-module $M$ is quasi-injective, if for any monomorphism $g$ of $A$-module $N$ into $M$ and for any morphism $f$ of $N$ into $M$, there exists an endomorphism $h$ of $M$ such: $f=h \circ g$.

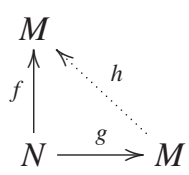

Definition 9 Let us considering two chains complexes of $A$-modules: $C$ and $E$. $C$ is said to be quasi-injective, if for any monomorphism $g$ of $E$ into $C$ and for any chain map $f$ of $E$ into $C$, there exists a chain map $h$ of $C$ into $C$ verifying $f=h \circ g$.

Theorem 3 Given $C: \ldots \rightarrow C_{n+1} \stackrel{d_{n+1}}{\rightarrow} C_{n} \stackrel{d_{n}}{\rightarrow} C_{n-1} \rightarrow \ldots$ a chain complex of A-modules. $C$ is quasi-injective, if and only if for all $n \in \mathbb{Z}, C_{n}$ a quasi-injective A-module.

Proof. Suppose that $R$ is quasi-injective chain complex and $f: M \rightarrow N$ a monomorphism of $A-$ modules and $\phi_{n}$ : $M \rightarrow R_{n}$ a morphism of $A$ - modules.

Considering $S$ and $R$ two chains complexes of $A$ - modules and $\alpha$ a chain of $S$ into $R$ such:

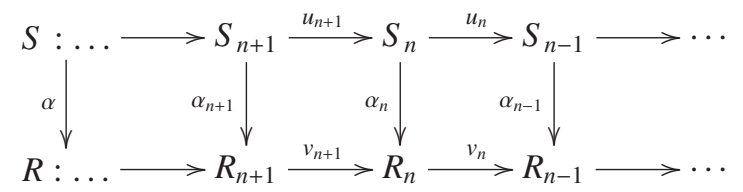

where for all $n \in \mathbb{Z} . S_{n}=M$ and $u_{n}=I d_{M}$ with $R_{n}=N$ and $v_{n}=I d_{N} \alpha_{n}$ is a monomorphism of $A-$ modules, for all $n \in \mathbb{Z}$.

Let $\phi$ a chain map of $S$ into $R$ such:

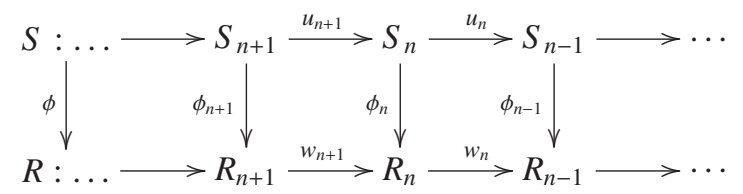

where for any morphismes $\phi_{k}: M \rightarrow R_{k}$ of $A$-modules. 
Given that $R$ is quasi-injective then it exists $\psi$ a chain map:

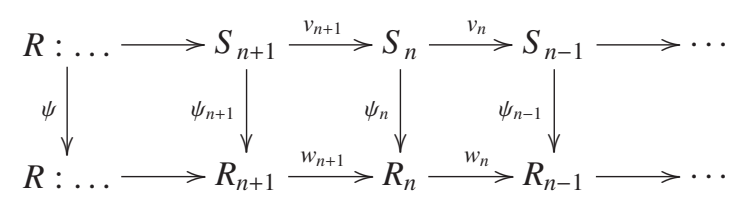

such: $\psi \circ \alpha=\phi$ hence $\psi_{n} \circ f=\phi_{n}$. Then it exists $\psi_{n}: R_{n} \rightarrow R_{n}$ such $\psi_{n} \circ f=\phi_{n}$ then $R_{n}$ is quasi-injective.

Reciprocally suppose that for all $n \in \mathbb{Z}, R_{n}$ is an $A$-quasi-injective module and let us prove that $R$ quasi-injective chain complex.

Let be $\gamma$ a monomorphism of chains complexes:

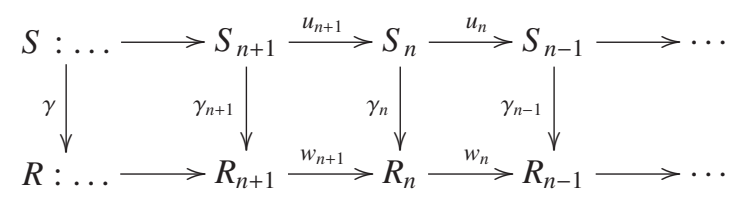

Considering $\beta$ a chain map of complexes such:

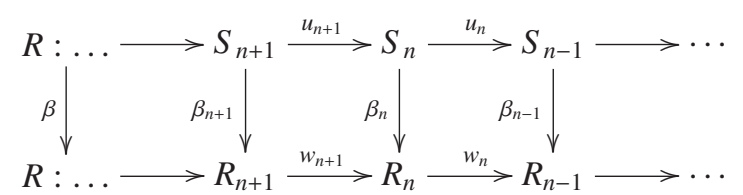

Then given for all $n \in \mathbb{Z}, R_{n}$ is quasi- injective and $\gamma_{n}$ is a monomorphism of $A$-modules hence it exists $\lambda_{n}: R_{n} \rightarrow R_{n}$ verifying $\beta_{n}=\lambda_{n} \circ \gamma_{n}$.

Let be $\lambda$ such:

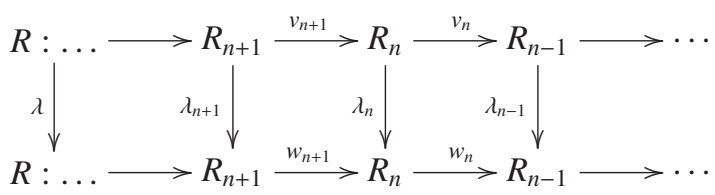

Let us demonstrate $\lambda$ is a chain map:

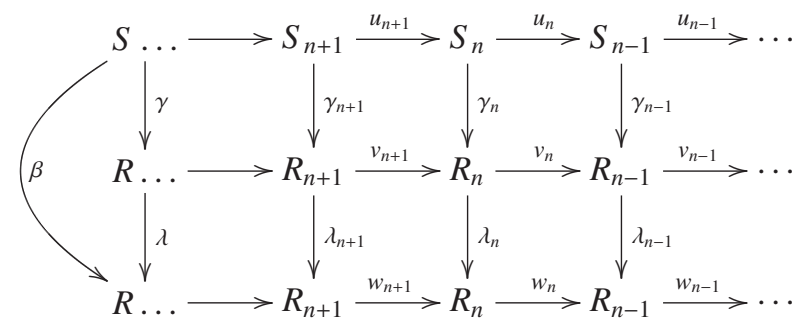

It is enough that: $w_{n+1} \circ \lambda_{n+1}=\lambda_{n} \circ v_{n+1}$.

We have: $w_{n+1} \circ \beta_{n+1}=\beta_{n} u_{n+1}$ but $\beta$ is chain map, because $\beta_{n}=\lambda_{n} \circ \gamma_{n}$. Then $w_{n+1} \circ\left(\lambda_{n+1} \circ \gamma_{n+1}\right)=\left(\lambda_{n} \circ \gamma_{n}\right) \circ u_{n+1}$. Therefore $\left(w_{n+1} \circ \lambda_{n+1}\right) \circ \gamma_{n+1}=\lambda_{n} \circ\left(\gamma_{n} \circ u_{n+1}\right)$. So $\left.\left(w_{n+1}\right) \circ \lambda_{n+1}\right) \circ \gamma_{n+1}=\lambda_{n} \circ\left(v_{n+1} \circ \gamma_{n+1}\right)$. Finally $\left(w_{n+1} \circ \lambda_{n+1}\right) \circ \gamma_{n+1}=$ $\left(\lambda_{n} \circ v_{n+1}\right) \circ \gamma_{n+1}$. But $\gamma_{n+1}$ is a monomorphism of $A$-modules then $w_{n+1} \circ \lambda_{n+1}=\lambda_{n} \circ v_{n+1}$, for all $n \in \mathbb{Z}$. so $\lambda$ is achain map.

Let us verify that: $\lambda \circ \gamma=\beta$. We know that for all $n \in \mathbb{Z}, \beta_{n}=\lambda_{n} \circ \gamma_{n}$ with $\beta=\left(\beta_{n}\right), \gamma=\left(\gamma_{n}\right)$ and $\lambda=\left(\lambda_{n}\right)$ so $\lambda \circ \gamma=\beta$. That prove $R$ is a quasi-injective complex.

Theorem 4 Given $C$ a chain complex of A-modules. If $C$ is quasi-injective and strongly hopfian, then $C$ is strongly cohop fian.

Proof. Suppose that $C$ is quasi-injective and strongly hopfian, then $C_{n}$ is quasi-injective using Theorem 3 and $\left(\operatorname{Im} f_{n}^{k}\right)$ stabilizing this implies that $C_{n}$ is quasi-injective and $\left(k e r f^{k}\right)$ stabilizes, so $C$ is strongly cohopfian. 


\subsection{Quasi Projective Chain Complex}

Definition 10 An $A$-module $P$ is said to be quasi-projective if for any $A$-module $N$ and any epimorphism $\pi: P \rightarrow N$ and any homomorphism $\phi: P \rightarrow N$, here exists an endomorphism $\psi: P \rightarrow P$ such $\pi \circ \psi=\phi$ illustrated by the following commutative diagramm:

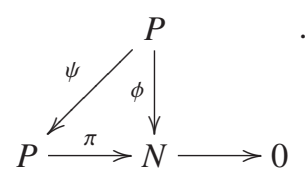

Definition 11 Given $C$ and $E$ two chains complexes of $A$-modules. $C$ is said to be quasi-projective chain complex if for any epimorphism: $C \rightarrow E$ and for any morphism $f: C \rightarrow E$, there exisrsa chain map $h: C \rightarrow C$ verifying: $f=g \circ h$, illustrated by the following commutative diagramm:

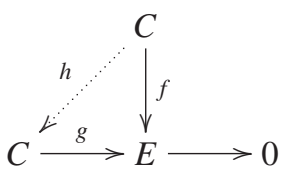

Theorem 5 Let $C: \ldots \rightarrow C_{n+1} \stackrel{d_{n+1}}{\rightarrow} C_{n} \stackrel{d_{n}}{\rightarrow} C_{n-1} \rightarrow \ldots$ a chain complex of A-modules. $C$ is quasi-projective if and only if for all $n \in \mathbb{Z}, C_{n}$ is a quasi-projective module.

Proof. Suppose that $E$ is quasi-projective.

Considering $f: N \rightarrow M$ an epimorphism of $A$-modules and $\phi_{n}: E_{n} \rightarrow M$ a morphisme of $A$ - modules.

Let $S$ and $E$ two chains complexes and $\alpha$ a chain map of $E$ into $S$ such:

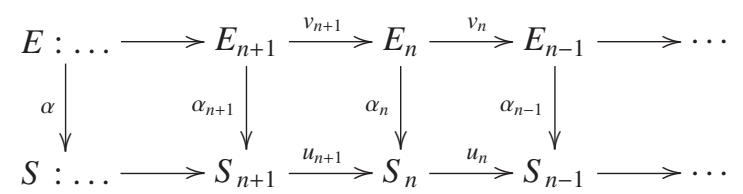

where $E_{n}=M$ et $v_{n}=I d_{M} . S_{n}=N$ and $u_{n}=I d_{N}$.

Given $\alpha_{n}$ an epimorphism of $A$ - modules. Let $\phi$ a chain of $E$ into $S$ verifying:

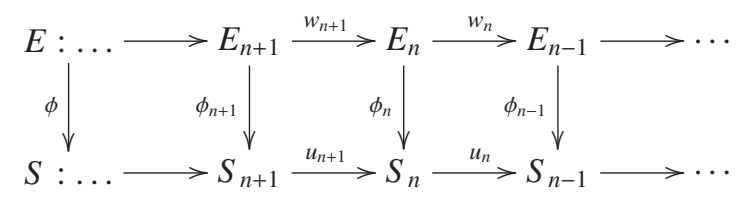

For any morphisms of $A$-modules $\phi_{k}: E_{k} \rightarrow M$.

Given $E$ is quasi-projective then it exists a chain map $\psi$ such:

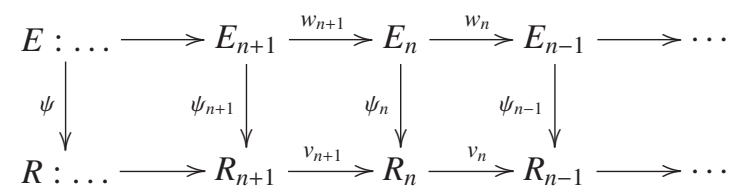

where: $\alpha \circ \psi=\phi$ so for all $n \in \mathbb{Z}, \alpha_{n} \circ \psi_{n}=\phi_{n}$. Then it exists $\psi_{n}: E_{n} \rightarrow R_{n}$ such $f \circ \psi_{n}=\phi_{n}$. So $E_{n}$ is quasi-projective.

Reciprocally suppose that for all $n \in \mathbb{Z}, E_{n}$ is quasi-projective $A$ - and let us demonstrate that $E$ is quasi-projective chain complex.

Considering $\gamma$ an epimorphism such:

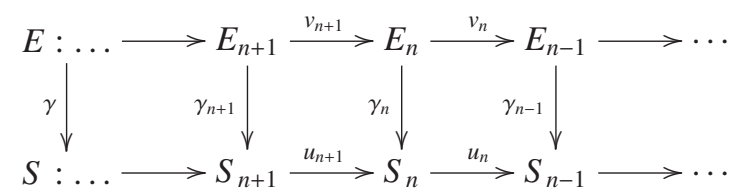


Let $\beta$ a chain map complex verifying:

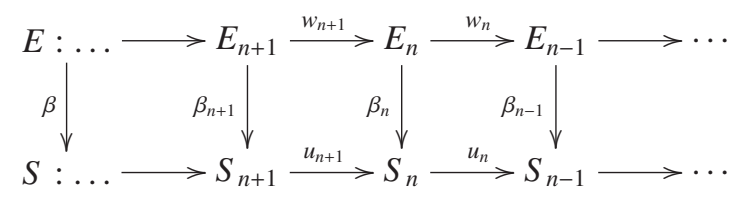

Then given for all $n \in \mathbb{Z}, E_{n}$ is projective and $\gamma_{n}$ is an epimorphism of $A$-modules so it exists $\lambda_{n}: E_{n} \rightarrow R_{n}$ verifying $\gamma_{n} \circ \lambda_{n}$.

Considering $\lambda$ such:

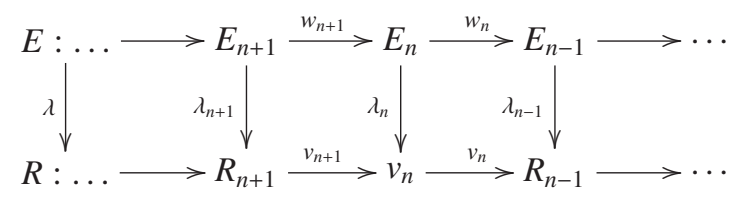

Let us demonstrate that $\lambda$ is a chain map

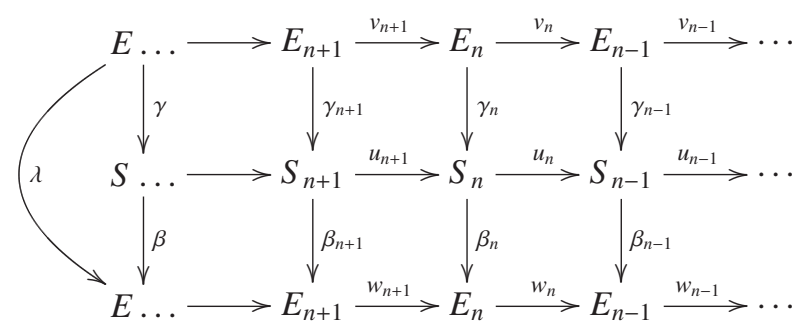

It is enough that: $\lambda_{n+1} \circ w_{n+1}=v_{n+1} \circ \lambda_{n}$. But $\beta_{n+1} \circ w_{n+1}=u_{n+1} \circ \beta_{n}$, because $\beta$ is a chain map.

Given $\gamma_{n} \circ \lambda_{n}=\beta_{n}$, then: $\gamma_{n+1} \circ \lambda_{n+1} \circ w_{n+1}=u_{n+1} \circ\left(\gamma_{n} \circ \lambda_{n}\right)$. So $\left(\gamma_{n+1} \circ \lambda_{n+1}\right) \circ w_{n+1}=u_{n+1} \circ\left(\gamma_{n} \circ \lambda_{n}\right)$. Then $\gamma_{n+1} \circ\left(\lambda_{n+1} \circ w_{n+1}\right)=\gamma_{n+1} \circ\left(v_{n+1} \circ \lambda_{n}\right)$. But $\gamma_{n+1}$ is an epimorphism hence $\lambda_{n+1} \circ w_{n+1}=v_{n+1} \circ \lambda_{n}$ What justifies that $\lambda$ is a chain complex.

Let us verify that: $\gamma \circ \lambda=\beta$. We know for all $n \in \mathbb{Z}, \beta_{n}=\gamma_{n} \circ \lambda_{n}$ so $\beta=\gamma \circ \lambda$. What justifies $E$ is a quasi-projective chain complex.

Theorem 6 Given $C$ a chain complex of A-modules. If $C$ is quasi-projective and strongly cohopfian then $C$ is strongly hopfian.

Proof. Suppose that $C$ is quasi-projective and strongly cohopfian, then $C_{n}$ is quasi-projective using Theorem 4 and $\left(k e r f_{n}^{k}\right)$ stabilizing this implies that $C_{n}$ is quasi-projective and $\left(k e r f^{k}\right)$ stabilizes so $C$ is strongly hopfian.

\subsection{Fitting Chains Complexes}

Definition 12 An $A$-module $M$ is said to be FITTING module if for any endomorphism $f$ of $M$, there exists a positive integer $n \geq 1$ such: $M=\operatorname{Kerf} f^{n} \oplus \operatorname{Im} f^{n}$.

Definition 13 A chain complex of $A$-modules is said to be FITTING chain complex if for any endomorphism $f$ of $C$, it exists $n \geq 1$ such $C=\operatorname{Kerf} f^{n} \oplus \operatorname{Im} f^{n}$.

Theorem 7 Considering $C$ a chain complex of A-modules such $C: \ldots \rightarrow C_{n+1} \stackrel{d_{n+1}}{\rightarrow} C_{n} \stackrel{d_{n}}{\rightarrow} C_{n-1} \stackrel{d_{n-1}}{\rightarrow} \ldots C$ is a FITTING chain complex if and only if for all $n \in \mathbb{Z}, C_{n}$ is A-FITTING module.

Proof. Suppose that $C$ is a FITTING chain complex of $A$-modules then it exists an positive integer $k$ such $C=$ $\operatorname{Kerf}^{k} \oplus \operatorname{Im} f^{k}$ donc $\operatorname{Kerf}^{k} \sqcap \operatorname{Im} f^{k}=0$ and $C=\operatorname{Kerf}^{k}+\operatorname{Im} f^{k}$ hence for all $n \in \mathbb{Z}, C_{n}=\operatorname{Kerf} f_{n}^{k}+\operatorname{Im} f_{n}^{k}$ so $C_{n}$ is strongly hopfian and strongly cohpfian then $C_{n}$ is a FITTING A-module. Reciprocally suppose that $C_{n}$ is an A-FITT ING module then $\left(\operatorname{Kerf} f_{n}^{k}\right)$ and $\left(\operatorname{Im} f_{n}^{k}\right)$ stabilizes so $\left(\operatorname{Kerf} f^{k}\right)$ stabilizes and $\left(\operatorname{Im} f^{k}\right)$ also. Which prove that $C$ is a FITTING chain complex.

Theorem 8 Any quasi-projective and strongly-hop fian or quasi-in jective and strongly-cohofian chain complex of A-modules is a Fitting chain complex.

Proof. Suppose that $C$ is quasi-projective and cohopfian then using the previous theorem we can say $C$ is hopfian, so $C$ is cohopfian and hopfian, then is a FITTING chain complex. 
Suppose that $C$ is quasi-injective and hopfian then using the previous theorem we can say $C$ is cohopfian, so $C$ is cohopfian and hopfian, then is a FITTING chain complex.

\section{References}

Anderson, F. W., \& Fuller, K. R. (1973). Rings and categories of modules. New York: Springer-Verlag.

Ben Maaouia Mohamed Ben Fraj. (1998). Dea-Faculté des Sciences et Techniques-UCAD-Dakar.

Ben Maaouia Mohamed Ben Fraj. (2003). Doctorat de 3ème cycle-Faculté des Sciences et Techniques-UCADDakar.

Faouzi, L. (2003). Hopficité et Co-hopficité des Anneaux et Modules Faculté des.

Haghany, A. (2001). Modules Whose Injective Endomorphismes are Essential. Journal of Algebra, 243, $765-779$. http://dx.doi.org/10.1006/jabr.2001.8851

Hiremath. (1986). Hopfian rings and co-Hopfian modules. Indian J. Pure and Appl. Math., 17, 895-900.

Hmaimou, A. (2007). Journal of algebra Generalized Fitting modules and rings, 308199-214. Sciences Tetouan.

Mukherjee, G. (1997). Hopfian And Co-Hopfian G-CW-Complexes. Proceedings of the American Mathematical Society, 125(4), 1229-1236. http://dx.doi.org/10.1090/S0002-9939-97-03778-7

Rotman, J. J. (1968). Notes on homological algebra. University of Illinois, Urbana.

Rotman, J. J. (1988). An Introduction to Algebraic Topology (Graduate Texts in Mathematics). http://dx.doi.org/10.1007/978-1-4612-4576-6

Rotman, J. J. (2000). An introduction to homological Algebra. New York: Academic Press.

Sanghare, M. (1993). Thèse d'état, Faculté des Sciences et Techniques. UCAD, Dakar.

Varadarajan, K. (1992). Hopfian and co-Hopfian objects. Publicacions Matematiques, 36, $293-317$. http://dx.doi.org/10.5565/PUBLMAT_36192_21

\section{Copyrights}

Copyright for this article is retained by the author(s), with first publication rights granted to the journal.

This is an open-access article distributed under the terms and conditions of the Creative Commons Attribution license (http://creativecommons.org/licenses/by/3.0/). 